\title{
Ophthalmomandibulomelic dysplasia
}

INSERM

\section{Source}

INSERM. (1999). Orphanet: an online rare disease and orphan drug data base.

Ophthalmomandibulomelic dysplasia. ORPHA:2741

Ophthalmomandibulomelic dysplasia is characterized by complete blindness due to corneal opacities, difficult mastication due to temporomandibular fusion and anomalies of the arms. 